\title{
The Windy Postman Problem on Series-Parallel Graphs
}

\author{
Francisco Javier Zaragoza Martínez ${ }^{\dagger}$ \\ Universidad Autónoma Metropolitana Azcapotzalco, Departamento de Sistemas, Av. San Pablo 180, Mexico City.
}

The windy postman problem is the NP-hard problem of finding the minimum cost of a tour traversing all edges of an undirected graph, where the cost of traversal of an edge depends on the direction. Given an undirected graph $G$, we consider the polyhedron $O(G)$ induced by the linear programming relaxation of a well-known integer programming formulation of the problem. We say that $G$ is windy postman perfect if $O(G)$ is integral. There exists a polynomialtime algorithm, based on the ellipsoid method, to solve the windy postman problem for the class of windy postman perfect graphs. Eulerian graphs and trees are windy postman perfect. By considering a family of polyhedra related to $O(G)$, we prove that series-parallel graphs are windy postman perfect, therefore solving a conjecture of Win (1987).

Keywords: windy postman problem, series-parallel graphs, integral polyhedra

\section{Contents}

1 Preliminaries

161

2 The Windy Postman Problem $r$

3 Windy Postman Ideal Graphs $r$

4 Conclusions $r$

\section{Preliminaries}

Let $G=(V, E)$ be an undirected graph, which may contain loops and parallel edges. The associated directed graph of $G$ is the directed graph $\vec{G}=\left(V, E^{+} \cup E^{-}\right)$obtained from $G$ by replacing each edge $e \in E$ by two oppositely oriented arcs $e^{+} \in E^{+}$and $e^{-} \in E^{-}$. For $S \subseteq V$ we define $\delta_{G}(S)$ to be the set of edges in $G$ with one end in $S$ and the other end in $\bar{S}$, and $\vec{\delta}_{G}(S)$ to be the set of arcs in $\vec{G}$ with tails in $S$ and heads in $\bar{S}$. We say that $e \in E$ crosses $S$ if $e \in \delta_{G}(S)$. We say that $G$ is even if $\left|\delta_{G}(v)\right|$ is even for all $v \in V$. A closed walk $W$ of $\vec{G}$ is a windy postman tour of $G$ if $W$ contains all vertices in $V$ and, for every $e \in E, W$ contains at least one of $e^{+}$or $e^{-}$. If $T$ is a set, $x \in \mathbb{R}^{T}$, and $S \subseteq T$ then $x(S)$ denotes the $\operatorname{sum} \sum_{s \in S} x_{s}$.

\footnotetext{
${ }^{\dagger}$ Partially supported by Universidad Autónoma Metropolitana Azcapotzalco grant 2270313 and CONACyT grant 69234. 


\section{The Windy Postman Problem}

Given an undirected graph $G$ with nonnegative costs $c$ on the arcs of $\vec{G}$, the windy postman problem consists of finding the minimum cost of a windy postman tour of $G$. This problem was proposed by Minieka (1979) and its decision version was shown to be NP-complete, even for planar inputs, by Guan (1984).

An integer programming formulation for the windy postman problem due to Win $(1987,1989)$ is:

$$
\begin{aligned}
\operatorname{WPP}(G, c) & =\min c^{\top} x \\
\text { subject to } & \\
x\left(\vec{\delta}_{G}(\bar{v})\right)-x\left(\vec{\delta}_{G}(v)\right) & =0 \text { for all } v \in V \\
x_{e^{+}}+x_{e^{-}} \geq & 1 \text { for all } e \in E \\
x_{e^{+}}, x_{e^{-}} & \geq 0 \text { for all } e \in E \\
x_{e^{+}}, x_{e^{-}} & \text {integral for all } e \in E .
\end{aligned}
$$

Let $P(G)$ be the convex hull of the feasible solutions to the integer program above, and let $Q(G)$ be the set of feasible solutions to its linear programming relaxation. Win $(1987,1989)$ proved that:

Theorem 1 (Win) Every extreme point $x$ of the polyhedron $Q(G)$ has components whose values are either $\frac{1}{2}$ or a nonnegative integer. Furthermore, $Q(G)$ is integral if and only if $G$ is even.

We can strengthen the linear programming relaxation of the windy postman problem by adding odd-cut constraints. Let $S \subseteq V$ be such that $\left|\delta_{G}(S)\right|$ is odd. Then, in any windy postman tour of $G$, at least one element of $\delta_{G}(S)$ must be used more than once. Therefore, the inequalities

$$
x\left(\vec{\delta}_{G}(S)\right)+x\left(\vec{\delta}_{G}(\bar{S})\right) \geq\left|\delta_{G}(S)\right|+1 \text { for all odd } S \subseteq V
$$

are valid for $P(G)$. Let $O(G)$ be the subset of $Q(G)$ that satisfies the odd-cut constraints (6).

We say that $G$ is windy postman perfect if the polyhedron $O(G)$ is integral. Equivalently, $G$ is windy postman perfect if $O(G)=P(G)$. Windy postman perfect graphs were studied extensively by Win (1987, 1989). Grötschel and Win (1992) proved that there exists a polynomial-time algorithm, based on the ellipsoid method, to solve the windy postman problem for the class of windy postman perfect graphs. This is a consequence of the equivalence of optimization and separation theorem and the fact that all the constraints (2), (3), (4), and (6) can be separated in polynomial time. By Theorem 1, even graphs are windy postman perfect. Win (1987) also proved that forests are windy postman perfect. Windy postman perfection is not closed under taking graph minors: $K_{5}$ is windy postman perfect, but $O\left(K_{4}\right)$ has fractional extreme points. Nevertheless, Win (1987) proposed operations that preserve windy postman perfection.

Theorem 2 (Win) Let $G, G_{1}, G_{2}$ be windy postman perfect graphs. Then:

1. Any subdivision of $G$ is windy postman perfect.

2. If $e \in E(G)$, then $G$ / $e$ is windy postman perfect.

3. If e, $f \in E(G)$ are parallel, then $G \backslash\{e, f\}$ is windy postman perfect. 
4. If $v_{1} \in V\left(G_{1}\right)$ and $v_{2} \in V\left(G_{2}\right)$, then the undirected graph $G_{3}$ obtained by identifying the vertices $v_{1}$ and $v_{2}$ is windy postman perfect.

We observe that the class of even undirected graphs is closed under each of these four operations, and that the same is true for the class of undirected forests. Another class of undirected graphs that has this property is the class of series-parallel undirected graphs. Win (1987) conjectured that these are also windy postman perfect. In what follows, we prove a statement stronger than Win's conjecture.

\section{Windy Postman Ideal Graphs}

Let $G=(V, E)$ be an undirected graph, let $l \in \mathbb{Z}_{+}^{E}$, and let $b \in \mathbb{Z}^{V}$ with $b(V)=0$. We say that $S \subseteq V$ is an odd set if $b(S)+l\left(\delta_{G}(S)\right)$ is odd. Let $\vec{G}=\left(V, E^{+} \cup E^{-}\right)$be the associated directed graph of $G$, and let $O(G, l, b)$ be the set of feasible solutions to the system

$$
\begin{aligned}
x\left(\vec{\delta}_{G}(\bar{v})\right)-x\left(\vec{\delta}_{G}(v)\right) & =b_{v} \text { for all } v \in V \\
x_{e^{+}}+x_{e^{-}} & \geq l_{e} \text { for all } e \in E \\
x\left(\vec{\delta}_{G}(S)\right)+x\left(\vec{\delta}_{G}(\bar{S})\right) & \geq l\left(\delta_{G}(S)\right)+1 \text { for all odd } S \subseteq V \\
x_{e^{+}}, x_{e^{-}} & \geq 0 \text { for all } e \in E .
\end{aligned}
$$

We say that $G$ is windy postman ideal if the polyhedron $O(G, l, b)$ is integral for all possible choices of $l$ and $b$. Observe that windy postman ideal graphs are windy postman perfect. We prove that windy postman ideal graphs are precisely the series-parallel graphs, proving Win's conjecture as a consequence. In contrast to windy postman perfection, windy postman ideality is closed under taking graph minors.

Theorem 3 Let $G=(V, E)$ be a windy postman ideal undirected graph, and let $e \in E$. Then $G \backslash e$ and $G /$ e are also windy postman ideal.

Proof. Let $u, v$ be the ends of $e$. Let $l^{\prime} \in \mathbb{Z}_{+}^{E \backslash e}$, and let $b^{\prime} \in \mathbb{Z}^{V(G / e)}$ with $b^{\prime}(V(G / e))=0$. Let $l \in \mathbb{Z}_{+}^{E}$ and $b \in \mathbb{Z}^{V}$ be defined by $l_{f}=l_{f}^{\prime}$ for all $f \in E \backslash e$ and $l_{e}=0$, and $b_{w}=b_{w}^{\prime}$ for all $w \in V \backslash\{u, v\}$, $b_{u}=b_{e}^{\prime}$, and $b_{v}=0$. Since $G$ is windy postman ideal, $O(G, l, b)$ is integral. Since $O\left(G / e, l^{\prime}, b^{\prime}\right)$ is the projection of $O(G, l, b)$ onto $x_{e^{+}}=0$ and $x_{e^{-}}=0$, it is also integral. Hence, $G / e$ is windy postman ideal.

Let $l^{\prime} \in \mathbb{Z}_{+}^{E \backslash e}$, and let $b^{\prime} \in \mathbb{Z}^{V}$ with $b^{\prime}(V)=0$. Define $l \in \mathbb{Z}_{+}^{E}$ by $l_{f}=l_{f}^{\prime}$ for all $f \in E \backslash e$ and $l_{e}=0$. Since $G$ is windy postman ideal, $O\left(G, l, b^{\prime}\right)$ is integral. Since $O\left(G \backslash e, l^{\prime}, b^{\prime}\right)$ is a face of $O\left(G, l, b^{\prime}\right)$, it is also integral. Hence, $G \backslash e$ is windy postman ideal.

Let $x \in O(G, l, b)$, and let $e \in E$. We say that $e$ is integral if both $x_{e^{+}}$and $x_{e^{-}}$are integral, and we say that $e$ is fractional otherwise. We say that $e$ is tight if $x_{e^{+}}+x_{e^{-}}=l_{e}$.

Lemma 4 Let $G=(V, E)$ be a minor minimal, non windy postman ideal undirected graph. Let $b \in \mathbb{Z}^{V}$ and $l \in \mathbb{Z}_{+}^{E}$ be such that $O(G, l, b)$ is not integral, and let $x$ be one of its fractional extreme points. If $e \in E$ is integral, then $x_{e^{+}}+x_{e^{-}}=l_{e}+1$.

The following two lemmas imply that we only need to consider 2-vertex-connected undirected graphs. 
Lemma 5 Let $G=(V, E)$ be an undirected graph, and let $G_{1}, \ldots, G_{k}$ be its connected components. Let $b \in \mathbb{Z}^{V}$ and $l \in \mathbb{Z}_{+}^{E}$. For every $1 \leq i \leq k$, let $b^{i}$ and $l^{i}$ be the restrictions of $b$ and $l$ to $G_{i}$. If $O\left(G_{i}, l^{i}, b^{i}\right)$ is integral for all $1 \leq i \leq k$, then $O(G, l, b)$ is also integral. Hence, $G$ is windy postman ideal if and only if $G_{i}$ is windy postman ideal for all $1 \leq i \leq k$.

Lemma 6 Let $G=(V, E)$ be an undirected graph with a cut vertex $v$, and let $G_{1}=\left(V_{1}, E_{1}\right)$ and $G_{2}=\left(V_{2}, E_{2}\right)$ be the partition of $G$ induced by $v$. Let $b \in \mathbb{Z}^{V}$ and $l \in \mathbb{Z}_{+}^{E}$. For $i \in\{1,2\}$, let $b^{i}$ and $l^{i}$ be the restrictions of $b$ and $l$ to $G_{i}$, except $b_{v}^{1}=b\left(V_{2}\right)$ and $b_{v}^{2}=b\left(V_{1}\right)$. Then $O(G, l, b)$ is integral if and only if $O\left(G_{1}, l^{1}, b^{1}\right)$ and $O\left(G_{2}, l^{2}, b^{2}\right)$ are integral. Hence, $G$ is windy postman ideal if and only if $G_{1}$ and $G_{2}$ are also windy postman ideal.

Now we state our characterization of windy postman ideal graphs.

Theorem 7 An undirected graph $G$ is windy postman ideal if and only if $G$ is series-parallel.

Sketch of proof. Since $O\left(K_{4}\right)$ is not integral, it follows that windy postman ideal graphs must be series-parallel. Let $G=(V, E)$ be a minor minimal, non windy postman ideal series-parallel graph. By Lemmas 5 and 6, we can assume that $G$ is 2-vertex-connected. We can verify that all series-parallel graphs with at most two vertices are windy postman ideal. Hence, we can assume that $G$ has two edges in parallel or two edges in series. Let $\vec{G}=\left(V, E^{+} \cup E^{+}\right)$be the associated directed graph of $G$, let $l \in \mathbb{Z}_{+}^{E}$, and let $b \in \mathbb{Z}^{V}$ with $b(V)=0$. For a contradiction, assume that $x$ is a fractional extreme point of $O(G, l, b)$.

Parallel case. Assume first that $G$ has two parallel edges $e$ and $f$, with ends $u$ and $v$. Let $H=(V, F)$ be the undirected graph obtained from $G$ by replacing edges $e$ and $f$ by a single edge $g$, and let $\vec{H}=$ $\left(V, F^{+} \cup F^{-}\right)$be its associated directed graph. We can assume that $e^{+}, f^{+}$, and $g^{+}$are oriented from $u$ to $v$, that $e^{-}, f^{-}$, and $g^{-}$are oriented from $v$ to $u$, and that all other arcs of $\vec{G}$ and $\vec{H}$ are oriented consistently. Define $l^{\prime} \in \mathbb{Z}_{+}^{F}$ by $l_{h}^{\prime}=l_{h}$ if $h \neq g$, and $l_{g}^{\prime}=l_{e}+l_{f}$. Note that $(G, l, b)$ and $\left(H, l^{\prime}, b\right)$ have the same odd sets.

Define $x^{\prime} \in \mathbb{Q}_{+}^{F^{+} \cup F^{-}}$by $x_{a}^{\prime}=x_{a}$ if $a \notin\left\{g^{+}, g^{-}\right\}, x_{g^{+}}^{\prime}=x_{e^{+}}^{\prime}+x_{f^{+}}^{\prime}$, and $x_{g^{-}}^{\prime}=x_{e^{-}}^{\prime}+x_{f^{-}}^{\prime}$, and observe that $x^{\prime} \in O\left(H, l^{\prime}, b\right)$. Assume first that $x^{\prime}$ is integral. In each of the two cases $x_{e^{-}}$is integral and $x_{e^{-}}$is fractional we can construct vectors $y \neq z$ such that $y, z \in O(G, l, b)$ and $x=\frac{1}{2}(y+z)$, contradicting the choice of $x$.

It follows that $x^{\prime}$ is fractional. Since $H$ is a minor of $G, O\left(H, l^{\prime}, b\right)$ is integral. Hence, there exist distinct vectors $y^{\prime}, z^{\prime} \in O\left(H, l^{\prime}, b\right)$ such that $x^{\prime}=\frac{1}{2}\left(y^{\prime}+z^{\prime}\right)$. We may choose $y^{\prime}, z^{\prime}$ so that $\left\|y^{\prime}-z^{\prime}\right\|$ is arbitrarily small. In each of the cases (a) neither $e$ nor $f$ is tight and (b) $e$ is tight, we can construct vectors $y \neq z$ (using $y^{\prime}$ and $z^{\prime}$ ) such that $y, z \in O(G, l, b)$ and $x=\frac{1}{2}(y+z)$, contradicting the choice of $x$.

Series case. Now assume that $G$ has two edges $e$ and $f$ in series, with ends $u$ and $v$, and $v$ and $w$, respectively. Using Lemma 4 and that $G$ is 2-edge-connected, we can prove that there are fractional edges in $E \backslash\{e, f\}$. Let $S^{e}$ be the set of all odd sets crossed by $e$, and let

$$
s_{e}=\min \left\{x\left(\vec{\delta}_{G}(S)\right)+x\left(\vec{\delta}_{G}(\bar{S})\right)-l\left(\delta_{G}(S)\right)-1: S \in S^{e}\right\} .
$$

Define $S^{f}$ and $s_{f}$ in a similar way. As above, by considering the minors $G /\{e, f\}$ and $G / e$, we can show that $s_{e}=s_{f}=0$. Using Lemma 4 we can show that $\{v\}$ must be an odd set. Let $t_{v}=$ $x_{e^{+}}+x_{e^{-}}+x_{f^{+}}+x_{f^{-}}-l_{e}-l_{f}-1$. Let $T^{e}$ be the set of all odd sets crossed by $e$, except for $\{v\}$ and its complement, let

$$
t_{e}=\min \left\{x\left(\vec{\delta}_{G}(T)\right)+x\left(\vec{\delta}_{G}(\bar{T})\right)-l\left(\delta_{G}(T)\right)-1: T \in T^{e}\right\},
$$


and let $T_{e} \in T^{e}$ achieve this minimum. Define $T^{f}, t_{f}$, and $T_{f}$ in a similar way. Since $s_{e}=s_{f}=0$, it follows that either $t_{v}=0$, or $t_{v}>0$ and $t_{e}=t_{f}=0$. As above, we can deal with these last two cases considering the minors $G / e$ and $G /\{e, f\}$, respectively.

\section{Conclusions}

Recall that if $G=(V, E)$ is not series-parallel, then it contains a subdivision $K=(W, F)$ of $K_{4}$. Define the vector $l \in \mathbb{Z}_{+}^{E}$ by $l_{e}=1$ if $e \in F$, and $l_{e}=0$ otherwise. Since $O\left(K_{4}\right)$ is not an integral polyhedron, it follows that $O(G, l, 0)$ is also not an integral polyhedron.

Corollary 8 Let $G=(V, E)$ be an undirected graph. Then $G$ is series-parallel if and only if the polyhedron $O(G, l, 0)$ is integral for all $l \in\{0,1\}^{E}$.

We also obtain Win's conjecture as an easy corollary.

Corollary 9 If $G$ is an undirected series-parallel graph, then $G$ is windy postman perfect.

Since the mixed postman problem can be seen as a special case of the windy postman problem, it also has a polynomial-time algorithm for the class of series-parallel graphs, a result of Fernandes et al. (2003).

It is possible to verify that $K_{3,3}$ is windy postman perfect. Using Theorems 1,2 , and 7 , we can extend the class of undirected graphs known to be windy postman perfect.

Theorem 10 All graphs in the class F constructed as follows are windy postman perfect: (a) All graphs whose connected components are even, series-parallel, or $K_{3,3}$ are in $F$, and (b) any graph obtained from graphs in $F$ by performing any of the operations described in the statement of Theorem 2 is in $F$.

A graft is a pair $(G, T)$ where $G=(V, E)$ is an undirected graph and $T \subseteq V$ with $|T|$ even. Let $l \in \mathbb{Z}_{+}^{E}$ and let $b \in \mathbb{Z}^{V}$ with $b(V)=0$. We say that the pair $(l, b)$ is valid for $(G, T)$ if for every $v \in V$, $b_{v}+l\left(\delta_{G}(v)\right)$ is odd if and only if $v \in T$. We say that $(G, T)$ is windy postman perfect if $O(G, l, b)$ is integral for each valid pair $(l, b)$. We have proved that windy postman perfection of grafts is closed under taking graft minors, therefore generalizing Theorem 3, and we have found two excluded minors for this property. We believe that these are the only two excluded minors for windy postman perfection of grafts. A proof of this statement would give rise to a common generalization of Theorems 1 and 7.

\section{Acknowledgements}

I would like to thank Bill Cunningham, Joseph Cheriyan, Bertrand Guenin, and Jim Geelen for their insightful comments and for their encouragement during my stay at the University of Waterloo.

\section{References}

C. Fernandes, O. Lee, and Y. Wakabayashi. The minimum cycle cover and the chinese postman problems on mixed graphs with bounded tree width. Available at http://www. ime. usp.br/ yw/, 2003.

M. Grötschel and Z. Win. A cutting plane algorithm for the windy postman problem. Math. Programming, 55(3, Ser. A):339-358, 1992.

M. G. Guan. On the windy postman problem. Discrete Appl. Math., 9(1):41-46, 1984. 
E. Minieka. The Chinese postman problem for mixed networks. Management Sci., 25(7):643-648, 1979.

Z. Win. Contributions to Routing Problems. PhD thesis, Universität Augsburg, Germany, 1987.

Z. Win. On the windy postman problem on Eulerian graphs. Math. Programming, 44(1, (Ser. A)):97-112, 1989. 